\title{
Philosophiques
}

\section{L'individuation selon Brentano}

\section{Daniel Schultess}

Volume 26, numéro 2, automne 1999

La critique de la raison en Europe centrale

URI : https://id.erudit.org/iderudit/004981ar

DOI : https://doi.org/10.7202/004981ar

Aller au sommaire du numéro

Éditeur(s)

Société de philosophie du Québec

ISSN

0316-2923 (imprimé)

1492-1391 (numérique)

Découvrir la revue

\section{Citer cet article}

Schultess, D. (1999). L'individuation selon Brentano. Philosophiques, 26(2),

219-230. https://doi.org/10.7202/004981ar

\section{Résumé de l'article}

Brentano, expert et continuateur de l'ontologie aristotélicienne, traite du problème métaphysique de l'individuation dans un petit nombre d'écrits (réunis dans le recueil intitulé Kategorienlehre ). Il retient pour ce problème une solution ordinairement rejetée, l'individuation par le lieu (qui serait de l'ordre de l'accident dans la perspective d'Aristote et comme tel impropre à fournir le principe de l'individuation ). À ce titre, il occupe une position originale. La notion de lieu, cependant, tout comme plus généralement la notion de l'accident, ressort tout à fait transformée des analyses de Brentano : le lieu devient une détermination substantielle, non accidentelle, des corps. Dans des écrits tardifs, Brentano étend son analyse du lieu à une cosmologie qui n'admet qu'une seule substance corporelle étendue, dont les corps particuliers sont les accidents.
Ce document est protégé par la loi sur le droit d'auteur. L'utilisation des services d'Érudit (y compris la reproduction) est assujettie à sa politique d'utilisation que vous pouvez consulter en ligne.

https://apropos.erudit.org/fr/usagers/politique-dutilisation/ 


\title{
L'individuation selon Brentano
}

DANIEL SCHULTESS

Université de Neuchâtel

Daniel.Schultess@lettres.uninen.ch

\begin{abstract}
RÉSUMÉ. - Brentano, expert et continuateur de l'ontologie aristotélicienne, traite du problème métaphysique de l'individuation dans un petit nombre d'écrits (réunis dans le recueil intitulé Kategorienlehre). II retient pour ce problème une solution ordinairement rejetée, l'individuation par le lieu (qui serait de l'ordre de l'accident dans la perspective d'Aristote et comme tel impropre à fournir le principe de l'individuation ). À ce titre, il occupe une position originale. La notion de lieu, cependant, tout comme plus généralement la notion de l'accident, ressort tout à fait transformée des analyses de Brentano : le lieu devient une détermination substantielle, non accidentelle, des corps. Dans des écrits tardifs, Brentano étend son analyse du lieu à une cosmologie qui n'admet qu'une seule substance corporelle étendue, dont les corps particuliers sont les accidents.
\end{abstract}

\begin{abstract}
Brentano, both expert and continuator of the Aristotelian ontology, devotes his attention to the metaphysical problem of individuation in a small number of writings collected under the title Kategorienlehre. For this problem, he adopts a solution which gets ordinarily rejected, individuation through place (place would be among the accidents within the Aristotelian perspective and as such unsuitable as the principle of individuation). Hence he occupies an original position on this issue. The notion of place, however, as well as the notion of accident more generally, gets completely transformed in the Brentanian analysis : place becomes not an accidental but a substantial determination. In some late writings, Brentano extends his analysis of place to a cosmology which admits a unique extended bodily substance, of which particular bodies are only the accidents.
\end{abstract}

\section{Introduction}

Dans l'histoire de la philosophie, on trouve dans la métaphysique plus d'un essai d'identifier le principe de l'individuation. II se trouve que Franz Brentano a développé des doctrines originales sur cette question. Ce sont surtout les analyses réunies par Alfred Kastil sous le titre Kategorienlehre qui le montrent ${ }^{1}$. Certes, la valeur de ces textes n'est pas soustraite à toute critique puisqu'ils n'ont pas été publiés par l'auteur, qu'ils résultent d'un découpage des manuscrits et qu'ils comportent des erreurs de transcription. M ais en attendant une édition plus fiable, il faut nous en contenter ; il faut dire aussi que sur cette question, les passages sont assez nombreux et convergents pour faire voir l'originalité de Brentano.

1. Brentano, Franz, Kategorienlehre, Kastil, A., dir., H ambourg, F. M einer (PhB 203), 1985 (dans les références ultérieures, nous donnons les datations des documents cités). N ous recourons aussi à Brentano, Franz, Philosophische Untersuchungen zu Raum, Z eit und Kontinuum, Kastil, A., St. Körner et Chisholm, R., dir., H ambourg, F. M einer (PhB 293), 1976, ainsi qu'à Brentano, Franz, Vom D asein G ottes, Kastil, A ., dir., H ambourg, F. M einer (PhB 210), 1980. 
M on plan sera le suivant. J 'examinerai d'abord un point préalable touchant à la solidarité au moins apparente de la question de l'individuation avec le réal isme des universaux. J e présenterai ensuite un tableau élémentaire de la façon dont le problème de l'individuation s'est historiquement mis en place. Je décrirai alors les idées de Brentano sur l'individuation par le lieu. Enfin, je considérerai les surprenantes extensions cosmologiques qu'il a cru pouvoir donner à sa problématique du lieu.

\section{L'individuation et le réalisme des universaux}

D'abord, en quoi consiste le problème de l'individuation? II s'agit, sous l'angle métaphysique, de rendre compte (commel'ont bien fait apparaître les travaux de Jorge J.E. G racia ${ }^{2}$ ) de la non-instantiabilité ou de l'incommunicabilité de l'individu : à la différence del'universel, qui peut se communiquer à une pluralité d'instances (par exemple l'universel « le bois » peut se communiquer à une multitude de pièces de bois ${ }^{3}$ ), l'individu (par exemple cette boiserie-ci ou l'une de ses parties) ne peut se communiquer à des instances.

En formulant le problème ainsi, on s'aperçoit qu'il a l'air de se poser au réaliste sur la question des universaux. Celui-ci demande ce qui doit en quelque sorte se surajouter à la nature universelle in re pour déterminer cette nature universelle à l'individualité dans ses différentes instances. Le réaliste présuppose donc, en soulevant ce problème, qu'il y a une nature universelle in re qui doive être rendue individuelle ou qui doive être complétée pour qu'on arrive à l'individu. Or, pour un anti-réaliste, ce présupposé est faux. Vu alors qu'il n'y a pas de nature universelle qui doive être rendue individuelle, y a-t-il encore lieu de rendre compte de la non-instantiabilité ou l'incommunicabilité de l'individu? $\mathrm{N}$ on, car celle-ci est en quelque sorte, pour l'anti-réaliste, un fait brut. II y a des individus, mais pas d'individuation. Exit notre problème.

On sera donc peut-être surpris que Brentano ait vu dans la non-instantiabilité de l'individu quelque chose dont le métaphysicien ait à rendre compte, vu son anti-réal isme qui s'affirme dans des formules comme « ce qui existe, existe individuellement ${ }^{4}$, ou bien : "Q ui croirait que, par un abstrait comme la nature du chat, on désigne quelque chose d'étant au sens propre, céderait à l'erreur et à l'absurdité. $»^{5}$

2. À côté des travaux proprement historiques, il faut citer Gracia, J.J.E., Individuality : An E ssay on the Foundations of M etaphysics, A lbany, NY, State University of N ew Y ork Press, 1988.

3. Brentano parle de «réduplication des individus» («Vervielfältigung der Individuen ») (Kategorienlehre, 30 septembre 1908, p. 34).

4. « Sicher ist, dass, was existiert, individuell existiert »(Brentano, Kategorienlehre, 30 septembre 1908, p. 39 (mais voir la suite)).

5. "Wer glaubte, dass durch ein Abstraktum wie Katzennatur etwas im eigentlichen Sinne Seiendes bezeichnet werde, würde einem Irrtum und einer Absurdität verfallen. » Brentano, Kategorienlehre, 4 février 1914, p. 61 ; cf. p. 60. 
Pourquoi Brentano agite-t-il un problème dont en apparence il aurait pu faire l'économie? Pour répondre à cette question préalable, il faut noter qu'il envisage d'abord l'universel à un autre niveau, celui des concepts. II observe que la pensée d'une chose peut être plus ou moins définie. II y a des présentations générales, comme la présentation d'une chose colorée, et des présentations plus spéciales, comme la pensée d'une chose rouge, et enfin, il y a la présentation de cette chose rouge individuelle (c'est un point qui devrait être discuté à part ${ }^{6}$ ). " N ous voyons donc que beaucoup de présentations nous laissent dans l'indétermination au sujet de la chose qui nous est présentée, mais non sur ce comme quoi elle nous est présentée. ${ }^{7}$

$\mathrm{N}$ ous avons donc la présentation d'une chose comme colorée, ou comme rouge, alors même que pour le reste, notre présentation est indéterminée et à ce titre générale. Pour notre problème de l'individuation, il s'agit alors de savoir ce qui devrait entrer dans la présentation générale d'une chose pour en fairela présentation individuelled'une chose. Parfois, Brentano situe effectivement la question de l'individuation sur ce plan conceptuel ${ }^{8}$.

$M$ ais il y a plus, car Brentano dénonce l'erreur nominaliste qui consiste à nier « que quelque chose puisse répondre à la présentation universelle ${ }^{9}$. Dans la chose, il y a donc "etwas», quelque chose, qui correspond (entsprechen) à la présentation universelle. Cet « etwas », ce quelque chose, n'est toutefois pas une partie qui ait à être complétée dans la chose ${ }^{10}$. O n ne parle de ce « etwas » comme d'un étant que dans un sens impropre ${ }^{11}$. Cette forme atténuée de réalisme ${ }^{12} n^{\prime}$ en laisse pas moins subsister le problème de l'individuation, ce « etwas » nerendant pas encore compte du caractère individuel des choses.

Voilà comment, en deux mots, je m'imagine qu'on peut expliquer la présence d'une doctrine de l'individuation chez Brentano. Cette entrée assez simple fait d'ailleurs en sorte qu'il aborde souvent cette question. II est curieux toutefois que la position qui fait que Brentano occupe une place originale dans le tableau des doctrines de l'individuation se présente surtout dans des textes des dernières années de la vie du philosophe (depuis 1915, et

6. Selon « Über Raum und Z eit » du 23 février 1917, « dans la perception visuelle, le point local ne peut donner d'individuation » (Brentano, Philosophische Untersuchungen zu Raum, Z eit und Kontinuum , p. 198-199; cf. p. 209). Cf. Brentano, Vom D asein Gottes, p. 419.

7. «So sehen wir denn, dass manche Vorstellungen uns über die Sache, die wir vorstellen in Unbestimmtheit lassen, nicht aber über das, als was wir sie vorstellen. » (Brentano, Kategorienlehre, 30 septembre 1908, p. 40 ; il souligne).

8. Ibid., p. 271 ; cf. plus loin.

9. «[Es irren] diejenigen, welche leugnen, dass dem universellen Vorstellen etwas entsprechen könne» (ibid., mars 1916, p. 21).

10. I bid., 4 février 1916, p. 60-61.

11. Ibid., p. 63.

12. Cf. Brentano, Philosophische U ntersuchungen zu Raum, Z eit und Kontinuum , p. 78. 
on sait qu'il ne vécut que jusqu'en mars 1917). Je m'intéresserai ici à cette dernière position.

\section{Cadre général}

La clause du besoin étant établie, voyons brièvement comment le problème de l'individuation s'est historiquement mis en place. Comme on le sait, Brentano continue à sa façon une métaphysique d'obédience aristotélicienne et dans une certaine mesure scolastique. M ême s'il l'a renouvelée, il en conserve certaines grandes lignes. Je pense notamment à la distinction de la substance et de l'accident - à laquelle nous reviendrons - , et au rôle prépondérant conféré à une intention de classifier les substances en genres et espèces.

C'est aussi en fonction de ces grandes lignes que s'est posé le problème de l'individuation dans I'histoire de la philosophie : plutôt que pour des événements, des processus, des états des choses, etc., il s'agit de savoir comment l'individuation se définit pour les substances et pour les accidents. En effet, tout comme il y a des substances universelles et des substances individuelles, il y a des accidents universels et des accidents individuels, comme on le voit notoirement au chapitre 2 des Catégories d'A ristote. Et, au plan des substances, il s'agit de savoir quel principe intervient pour déterminer les espèces spécialissimes à l'individualité (parfois, on dit que c'est l'individu lui-même qui est l'espèce spécialissime).

En ce qui concerne l'individuation de la substance, la scolastique avait en général refusé la thèse que nous pourrions assez spontanément envisager, et qui serait que ce sont les accidents (les lieux, les quantités et qualités, le temps, etc.) qui déterminent les espèces à l'individualité. $N$ ous verrons que Brentano lui-même rejoint cette attitude, quoique ce soit à vrai dire à travers un changement de l'extension des catégories de l'accident.

Avec les éléments que nous avons posés, nous arrivons à un tableau sommaire des doctrines de l'individuation, qui nous servira à situer Brentano. Certains acceptent l'individuation de la substance par les accidents (des auteurs platonisants et Leibniz). D'autres professent l'individuation de la substance indépendamment des accidents. Les tenants de cette seconde position disent que, là où on s'adresse aux substances matérielles, I'individuation peut se faire par la matière ou par la forme. Ces doctrines opposées principales ont leurs défenseurs: Thomas d'A quin pour la première ( $c$ 'est parce que la matière entre dans la composition des substances individuelles que celles-ci sont non communicables), J ean Duns Scot pour la seconde (c'est parce qu'une forme individuelle, dite parfois « haeccéité »- Brentano dit " hoccéité ${ }^{13}-$, entre dans la composition des substances individuelles que celles-ci sont non communicables ; on sait que Socrate est un bipède sans plumes ; son caractère bipède et son absence de plumes ou d'autres traits

13. Brentano, Kategorienlehre, p. 21, p. 148. 
généraux restant universels, c'est une forme individuelle, la socraticité, qui fait que Socrate ne se communique pas à des instances). N ous verrons que Brentano occupe une position distincte des deux précédentes.

\section{La dernière position de Brentano}

Dans son édition de la K ategorienlehre, Kastil a insisté sur la discontinuité de la position de Brentano concernant l'individuation. II y a dans cette insistance des aspects justifiés et d'autres qui le sont moins. ${ }^{14}$ |l est clair que Brentano a admis assez so uvent quel'individuation se faisait par la réunion d'une pluralité de déterminations qui, prises isolément, restent seulement spécifiques. « $M$ ais les deux séries de différences peuvent réaliser ce que J ean $D$ uns Scot a cherché dans une seule avec sa hoccéité. » ${ }^{15}$ L'individuation proviendrait de la pluralité des séries prédicamentales à l'intérieur du genre. Cellesci sont telles qu'ensemble elles définissent un et un seul individu. Brentano est alors dans la ligne de Leibniz. II critique autant A ristote et Thomas ${ }^{16}$ que J ean Duns Scot pour n'avoir pas vu la possibilité que l'individuation provienne de la pluralité des séries prédicamentales à l'intérieur d'un même genre.

M ais Brentano n'accepte pas cette position dans les années 1915-1917, pour la raison assez simple qu'une réunion de déterminations spécifiques continuerait, comme celles-ci, à ne pas être déterminée à l'individualité :

II est bien sûr clair qu'une détermination générique, s'ajoutant à la détermination spécifique, ne peut contribuer à l'individualisation. En effet, elle est toujours incluse dans la différence spécifique. $M$ ais l'adjonction d'une espèce spécialissime, qui n'est pas en elle-même individualisée, ne peut pas davantage donner à l'autre l'individuation. ${ }^{17}$

$C^{\prime}$ 'est sur cette base que Brentano en arrive à la position que nous devons discuter. II écrit par exemple : « la species specialissima est la déter-

14. Cf. ibid., p. 274-275. A. Kastil annote ce texte de 1916 de la façon suivante : « Damit wird die von Brentano früher vertretene Auffassung, wonach die Individuation der Vereinigung von lauter universellen Bestimmungen zu verdanken wäre, endgiltig verlassen. » (ibid., p. 387, n.34). Ce découpage historique des positions de Brentano est contestable, puisque dans les cours de W ürzburg et de Vienne, Vom D asein Gottes (1868-1891), il écrit : " So ist es hier der O rt, der die Individualität ausmacht. » (Brentano, Vom D asein G ottes, p. 418). Cf. aussi ibid., p. 430n.

15. «Allein beide Differenzreihen zusammen mögen das leisten, was Scotus mit seiner hocceitas in einer gesucht hat. » Brentano, Kategorienlehre, 1912/1913, p. 148. Cf. ibid., 30 septembre 1908, p. 37.

16. Ibid., p. 22, cf. aussi p. 105-106.

17. «Es ist nun klar, dass eine Gattungsbestimmung, zur Speziesbestimmung hinzukommend, nicht zur Individualisation beitragen kann. Ist sie doch immer in der spezifischen Differenz mitbeschlossen. Ebensowenig aber kann das $\mathrm{H}$ inzukommen einer species specialissima, die nicht in sich selbst individualisiert ist, zur andern ihr die Individuation geben. » (ibid., 1916, p. 274-275) 
mination spatiale ${ }^{18}$, ou bien affirme que $\ll$ le concept du local [...] individualise ${ }^{19}$. Cette position est donc :

(IL) le lieu est principe de l'individuation

De cette thèse principale (IL), il faut distinguer des thèses successives sur lesquelles elle s'appuie et qui se combinent dans la doctrine de Brentano concernant l'individuation. D ans ses manuscrits, notre auteur livre ces thèses dans un certain enchevêtrement. C'est pourquoi je m'essaie à une analyse dans laquelle je distingue la réalité du lieu $(R L)$, le caractère substantiel du lieu (LS) et son caractère individuel (LDI).

$R L$

D'abord, cette position ne se conçoit pas sans postuler la réalité du lieu.

$(R L)$ le lieu est quelque chose de réel ${ }^{20}$

Si le lieu est tenu pour individuateur des corps, il doit être tenu pour quelque chose de réel. II ne peut être ni idéal - la thèse de Brentano est antileibnizienne ou anti-relationnelle en ce qui concerneles lieux - , ni inexistant.

\section{$L S$}

Cela posé, on ne dit pas encore quelle sorte de réalité revient au lieu. Brentano s'avère ici original en ce que pour lui :

(LS) le lieu est d'ordre substantiel

Voici comment Brentano exprime cette thèse anti-aristotélicienne, mais aussi anti-leibnizienne :

On ne pourrait disputer que les différences de lieu sont des différences d'ordre substantiel, que si l'on parvenait à croire que cela soit accidentel pour quelque chose de se trouver en un lieu, ou d'être quelque chose de corporel, ce qu'on ne pourra pas prétendre à la légère. ${ }^{21}$

Ce qu'assure ici Brentano, c'est qu'on ne peut pas croireque pour quelque chose, il soit accidentel d'être en un lieu si cette chose est en un lieu (ou d'être un corps si cette chose est un corps).

18. «Species specialissima ist die räumliche Bestimmtheit » (ibid., 1916, p. 268).

19. «Der Begriff des Ö rtlichen, sow ohl wo er individualisiert... », ibid., 1916, p. 271 il faut souligner « er » et entendre « der Begriff des Ö rtlichen individualisiert »; cf. plus haut, paragraphe 1 .

20. Brentano, Philosophische U ntersuchungen zu Raum, Z eit und Kontinuum , p. 209.

21. « N ur dann könnte man bestreiten, dass die örtlichen Differenzen substanzielle seien, wenn man glauben könnte, dass es für etwas akzidentell sei, überhaupt an einem O rte sich zu befinden und etwas Körperliches zu sein, was nicht leicht jemand behaupten wird. » (Brentano, Kategorienlehre, 1916, p. 247). 
R appelons ici la doctrine de Brentano sur la substance et l'accident. O n part des différences au sein de la prédication comme avec les exemples suivants: "mon crayon est un corps», "mon crayon est rouge». Ce qu'indique le prédicat peut se situer dans le genre de la substance ou dans le genre de l'accident. Comment déterminer si I'on se trouve dans un cas ou dans l'autre? Cela se fait par le critère de la détachabilité. Si, comme avec « ...est rouge », le prédicat fait apparaître un sujet dont ce qu'indique le prédicat est détachable, on est dans le cas d'un prédicat de l'accident. S'il n'y a pas de tel sujet, on est dans le cas de la substance ( « ...est un corps », « ...est en un lieu »). Ce critère est classique, même s'il élimine déjà, pour rappel, les sottises de J ohn L ocke sur la substance. Brentano maintient alors que l'accident est ce qui enveloppe comme partie (als Teil einschliesst) une substance. Pour prendre des exemples psychologiques, l'accident, cen'est pas le vouloir, le jugement, l'amour, mais le voulant, le jugeant, l'amant. $N$ ous sommes tous des accidents! - à cette précaution près que les conditions d'identité des accidents et des substances ne sont pas les mêmes ${ }^{22}$.

Par ce critère, on voit que, dans la mesure où la localisation est sujet d'accidents détachables, mais ne renvoie pas elle-même à un sujet dont elle serait détachable, elle est dernier sujet ou substance : « [I]elocal, qui est sousjacent au qualitatif [...] est pour lui dernier sujet $\gg .{ }^{23} \mathrm{~N}$ ous arrivons donc au résultat suivant:

(LS') le lieu est dernier sujet, c'est-à-dire substance.

À ce stade de la discussion, on peut parler d'une dépendance libre des accidents à l'égard de la local isation comme dernier sujet. C'est en quelque sorte génériquement qu'il faut une localisation aux accidents.

Le temps est aussi une détermination substantielle en ce sens; pour le temps aussi, on rencontre une telle dépendance libre :

Cela peut surprendre que nous prenions les déterminations de temps et de lieu, qui appartiennent pourtant à des séries de spécification différentes, toutes deux comme des déterminations substantielles. Chez A ristote, cela serait inacceptable car, sel on lui, deux spécifications de séries différentes ne peuvent être substantielles l'une et l'autre. ${ }^{24}$

22. I bid., 30 septembre 1908, p. 37-38.

23. Das Örtliche, « welches dem Qualitativen unterliegend und ihm durch seine letzte spezifische Bestimmtheit die individuelle Differenz gebend als letztes Subjekt für sie erscheint » (ibid., 1916, p. 269).

24. «Auffallen mag, dass wir Zeit- und Ortsbestimmungen, die doch verschiedenen Serien der Spezifikation angehören, beide als substanzielle fassen. $\mathrm{N}$ ach A ristoteles ginge das nicht, nach ihm können zwei Bestimmungen verschiedener Serien nicht beide substanziell sein » (ibid., 1916, p. 248). 
Pour le temps, on ne pourra cependant aller au-delà d'une dépendance libre. Si on allait au-delà, l'individu ne pourrait conserver son identité dans le temps. ${ }^{25}$

$L D I$

Pour une doctrine de l'individuation par le lieu, il ne suffit pas que les choses soient dépendantes des localisations, au sens d'une dépendance libre. Ce serait trop faible. II faut noter maintenant que :

(LDI) le lieu possède la détermination individuelle

Le lieu est complètement déterminé et à ce titre il est individuel.

[J e reconnais] que celui-ci [le local comme sujet du coloré] est spécifiquement parfaitement déterminé et que dans cette complète détermination spécifique il est individualisé, que par conséquent lui-même ne peut avoir d'autre sujet, ce qui est le propre de la substance. ${ }^{26}$

Le lieu se distingue en cela des autres déterminations qui ne sont pas déterminées jusqu'à être individuelles. « Ainsi quelque chose qui a les déterminations du rouge et du chaud reste toujours dépourvu de détermination individuelle, et aussi quelque chose qui a les déterminations de l'acceptation et du désir d'un certain objet. Et ainsi la réunion d'autant de species specialissimae qu'on voudra ne conduira à aucune individuation, s'il ne se trouve pas que l'une d'entre elles inclue aussi, dans sa détermination spécifique, la détermination individuelle. ${ }^{27}$

$\mathrm{N}$ otons pour le contraste que dans la perspective de Leibniz, par exemple, le lieu ne pourrait pas être tenu pour individuellement déterminé :

Les parties du temps ou du lieu prises en elles-mêmes sont des choses idéales, ainsi elles se ressemblent parfaitement comme deux entités abstraites. $M$ ais il n'en est pas de même de deux uns concrets, ou de deux temps effectifs, ou de deux espaces remplis, c'est-à-dire véritablement actuels. ${ }^{28}$

25. Pour compléter l'articulation lieu/temps dans la théorie de l'individuation, il faut dire ceci : ce qui est quelque chose de réel, ce sont les lieux en un temps qui est présent; ce qui individue, c'est un lieu à ce temps-là ; le temps donc, tout en étant une détermination substantielle, ne contribue pas de la même façon à l'individuation, car on ne va pas au-delà de la dépendance générique.

26. « [Ich erkenne] dass dieses [das Örtliche als Subjekt von Farbigem] vollkommen spezifisch determiniert und in dieser vollen spezifischen Determination individualisiert ist, dass es also selbst kein anderes Subjekt haben kann, was eine Eigentümlichkteit der Substanz ist. » (ibid., 1916, p. 271).

27. « So ist ein Rotes-Warmes Bestimmtes immer noch ohne individuelle Bestimmung und ein einen gewissen Gegenstand Anerkennendes und Begehrendes ebenfalls. Und so würde denn die Vereinigung von noch so vielen species specialissimae zu keiner Individuation führen, wenn nicht eine derselben in ihrer spezifischen Differenzierung auch die individuelle Determination besässe. » (ibid., 1916, p. 275).

28. Correspondance Leibniz-Clarke, $5^{e}$ écrit de Leibniz, no.27. Cf. aussi Leibniz, N ouveaux Essais, II.xxvii.1. 
IL

C'est ainsi que nous arrivons à la thèse principale (IL) :

(IL) le lieu est principe de l'individuation

La réflexion qui doit valider ce point d'arrivée est modale :

Toute détermination appartient à l'unité de cette substance, qui ne peut pas complètement faire défaut et qui ne peut pas varier non plus sans que la substance ne devienne une substance individuellement distincte. ${ }^{29}$

Cela vaut en particulier pour le lieu. Brentano exprime ici la dépendance liée, c'est-à-dire à l'égard d'un lieu déterminé, pour les choses dans leur identité numérique.

$M$ ais comment prendre la formule « ne peut pas varier »? O n peut l'entendre, d'un côté, en un sens factuel que j'exprimerais ainsi :

(SF) chaque fois qu'un individu se trouve dans le lieu $A$ en $t$ et qu'un individu se trouve dans le lieu $B$ en $t+* t$, alors l'individu qui se trouve dans le lieu $A$ en t et l'individu qui se trouve dans le lieu $B$ en $t+* t$ sont numériquement distincts.

Dans cette interprétation, on ne peut en quel que sorte arracher la chose individuelle à son lieu salva individualitate. Cela peut faire sourire, mais on trouve un tel raisonnement chez Thomas d'A quin et chez J ean D uns Scot, qui I'opposent à la thèse de l'individuation par les accidents (notamment lelieu)..$^{30}$

$M$ ais (SF) paraît faux, car les changements de lieu seraient alors incompatibles avec l'unité numérique. Cela me semble constituer une réfutation de cette interprétation de la formule « ne peut pas varier ». Aussi celle-ci doitelle s'entendre plutôt dans un sens contrefactuel de « ne peut pas varier »:

(SC) si une certaine chose, qui occupe le lieu $A$ en $t$, occupait en $t$ un autre lieu $B$ que celui qu'elle occupe, elle ne serait pas le même individu dans le cas où elle occupe le lieu $\mathrm{A}$ et dans le cas où elle occupe le lieu $\mathrm{B}$.

La formule (SC) évite les embarras précédents, mais entraîne aussi une certaine forme de nécessité de la localisation : il est nécessaire à une certaine chose d'avoir la localisation qu'elle a. M ais on observera que c'est une nécessité seulement conditionnelle : pour la chose qui est au lieu A au temps $t$, il est nécessaire d'être au lieu $A$ en $t$; et il est impossible, pour des raisons non pas physiques mais logiques, d'être au lieu $B$ en $t$ (où les lieux $A$ et $B$ diffèrent). Parce qu'elle est conditionnelle, cette nécessité ne me semble avoir aucune conséquence déterministe. Celle-ci n'interviendrait que si la condition était supprimée : pour une chose individuelle donnée, il est nécessaire

29. «Jede Bestimmung gehört zu der Einheit dieser Substanz, welche nicht gänzlich entfallen und auch nicht variieren kann, ohne dass die Substanz eine individuell andere würde. » (Brentano, Kategorienlehre, 1916, S.248).

30. Thomas d'A quin, Expositio super librum Boethii de trinitate, q.4, a.2, paragraphe 7 ; J ean Duns Scot, O rdinatio II.iii.1, paragraphe 77. 
d'être au lieu $A$ en $t$ (où $t$ est un temps futur). $M$ ais la formule restreinte par une condition n'implique pas la formule non restreinte.

C ette réflexion peut être étendue au passé. Pour la chose qui a été au lieu $A$ au temps t (où t est un temps passé), il est nécessaire d'avoir été au lieu $A$ en $t$. $M$ ais cela n'a pas non plus de conséquence déterministe. En effet, avant le temps $t$, il n'a pas été nécessaire pour la chose d'avoir été au lieu $A$ en $t$.

II me semble que tels sont les points qui constituent le contenu de la doctrine de Brentano sur l'individuation. Je n'ai rien dit de la méthode d'une telle investigation, mais je ne crois pas devoir chercher à ce sujet un grand raffinement. II me semble que nous sommes invités à ratifier ces points dans la mesure où ils sont « évidents a priori » (« a priori einleuchtend») comme d'autres jugements de logique ou de métaphysique.

\section{Extensions cosmologiques}

Dans les doctrines de Brentano, les idées sur l'individuation doivent être mises en rapport avec des questions cosmologiques ${ }^{31}$.

\section{(a) l'impénétrabilité}

L'impénétrabilité des corps (ce que Leibniz appelait I'antitypie) repose, selon Brentano, sur leur principe d'individuation, qui fait que si deux corps coïncidaient par le lieu, ils cesseraient d'être deux. ${ }^{32}$

\section{(b) l'unicité de la substance étendue}

Brentano, on s'en souvient, dit que la substance est une partie de l'accident, la partie qui n'a pas de nouveau de partie. II ajoute que le lieu est substantiel en ce sens ; mais pour chaque corps qualitativement déterminé, ce lieu substantiel a des limites (le lieu, chez A ristote, est la limite immobile première de l'enveloppe ${ }^{33}$ ).

D ans une hypothèse cosmologique surprenante, Brentano étend sa notion du lieu substantiel et soutient qu'il y a une seule substance unifiée. ${ }^{34}$ Au lieu que l'accident enveloppe une substance qui sejuxtapose à d'autres au sein d'un système plus large des lieux (occupés ou non), l'accident n'enveloppe qu'une partie d'une substance unitaire. ${ }^{35}$ C ette substance, Brentano la

31. Sur ces relations, cf. la note de A. Kastil dans Brentano, Kategorienlehre, p. 392, n.8.

32. Cf. «Die Undurchdringlichkeit» Brentano, Philosophische Untersuchungen zu Raum, Z eit und Kontinuum, 1915, p. 183.

33. Cf. A ristote, Physique IV.4, 212a21-22.

34. Cf. le manuscrit «Die Natur der Körperwelt im Lichte der Kategorienlehre», Brentano, Kategorienlehre, 30 janvier 1915, p. 296-301.

35. «Die einheitliche körperliche Substanz », ibid., p. 300. Cf. aussi Brentano, Vom D asein G ottes, p. 421-422 ; Kastil, A., D ie Philosophie Franz Brentanos, Berne, Francke, 1951, p. 23. 
substitue à l'éther de la physique de la fin du XIX ${ }^{e}$ siècle, ce milieu dans lequel se manifestent les ondes électromagnétiques. ${ }^{36}$

Pourquoi étendre ce dont on parle au titre de substance (d'un lieu) en quelque sorte au système des lieux? C'est une question à propos de laquelle je suis un peu perplexe. Cette hypothèse résout le problème des lieux inoccupés. D'autre part, en suivant une suggestion de Kastil, on peut se dire que cette hypothèse élimine la difficulté de rendre compte de l'unité de l'espace : « Tout en étant un, il aurait pourtant des parties ; et cependant que dans ses différentes parties, il constituerait le fondement des qual ités au titre de sujet, il mettrait en accord la multiplicité des corps et l'unité de l'espace ${ }^{37}$.

Ce déplacement est exploré par Brentano dans ses diverses conséquences : les lois de la nature physique, au lieu d'être des lois qui concernent les substances corporelles, sont les lois qui concernent les accidents de la substance :

À la place de ce qu'auparavant on considérait comme substance de la matière corporelle viendraient des accidents qui, rattachés à la substance, se transféreraient d'une partie à l'autre. Les lois de la mécaniques se rapporteraient au dé placement et à leur maintien en repos ${ }^{38}$.

Le rapport âme-corps se comprend de la même façon, c'est-à-dire non pas comme rapport avec un corps propre qui soit lui-même substance, mais rapport avec les accidents d'une substance:

Q ue notre âme puisse être considérée en interaction avec les qualités inhérentes à la substance corporelle unifiée tout aussi bien qu'elle l'était auparavant avec les substances corporelles, c'est ce qu'il n'est pas nécessaire d'observer expressément ${ }^{39}$.

\section{Conclusion}

Brentano a élaboréune doctrine originale de l'individuation et du lieu. Parmi ceux qui ont pensé que la localisation joue un rôle dans la problématique de l'individuation, il a dégagé une perspective très attrayante pour que la localisation puisse vraiment se voir conférer un tel rôle.

36. «Der Gedanke der einheitlichen Substanz, die an die Stelle des Aethers träte » (Brentano, Kategorienlehre, p. 299).

37. K astil, D ie Philosophie Franz Brentanos: «W ie immer einer, so hätte er doch Teile und indem er seinen verschiedenen Teilen nach den Q ualitäten als Subjekt zugrunde läge, wäre die Vielheit der Körper mit der Einheit des Raumes im Einklang» (p. 185).

38. «An die Stelle vom dem, was man früher als Substanz der körperlichen M aterie betrachtete, träten A kzidentien, welche, an der Substanz haftend, von einem Teil auf den anderen sich übertrugen. A uf ihren Stellenwechsel und auf ihr ruhiges Beharren bezögen sich die Gesetze der M echanik. » (Brentano, Kategorienlehre, p. 298)

39. «Dass unsere Seele zu den der einheitlichen körperlichen Substanz inhärierenden Q ualitäten ganz ebenso in Wechselwirkung gedacht werden könnte wie früher zu den körperlichen Substanzen, braucht nicht ausdrücklich bemerkt zu werden. » (ibid., p. 300) 
En ce qui concerne le volet cosmologique, je pense que l'argument sur l'impénétrabilité est dénué de valeur parce que la non-impénétrabilité salva individualitate dont Brentano nous parle ne peut pas avoir de conséquences physiques. Celles-ci me semblent ne pouvoir se fonder que sur une interaction physique. En revanche, la théorie de l'étendue substantielle unique me paraît hardie et digne de recevoir davantage d'attention qu'elle n'en a reçue jusqu'ici. 